\title{
ON THE MINIMUM MODULUS OF TRIGONOMETRIC POLYNOMIALS
}

\author{
GEORGE BENKE
}

(Communicated by J. Marshall Ash)

\begin{abstract}
For all even integers $N$ greater than 2, a trigonometric polynomial $f_{N}(x)=\sum_{k=-N}^{N^{2}} a_{k} e^{i k x}$ satisfying $\left|a_{k}\right| \leq 1$ and $0.47 N \leq\left|f_{N}(x)\right| \leq N$ is constructed.
\end{abstract}

In this paper we are concerned with the problem of finding polynomials $P_{n}$ of degree $n$ whose coefficients are bounded by 1 and whose modulus $\left|P_{n}(z)\right|$ on $|z|=1$ exceeds $A\left\|P_{n}\right\|_{\infty}$, for some absolute constant $A>0$. This problem is part of the more general question, as expressed by Beller [Be]: "How close can we get to a situation where $P(z)$ is a polynomial of degree $n>0$ which, on the one hand, has coefficients of constant modulus, and on the other hand $|P(z)|$ is constant for $|z|=1$ ?" The question goes back to Littlewood [L] and has also been studied by Beller and Newman [BeN], Byrnes [By], Körner [Ko], and Kahane [Ka]. Problem 27 of Erdös [E] is also concerned with this topic. From the standpoint of existence, the question has been emphatically answered by Kahane [Ka] where he proves the following.

Theorem. There exists a sequence of polynomials

$$
P_{n}(z)=\sum_{m=1}^{n} a_{m, n} z^{m}
$$

with $\left|a_{m, n}\right|=1$ and a positive sequence $\varepsilon_{n}$ converging to 0 such that for all $|z|=1$ the following holds:

$$
\left(1-\varepsilon_{n}\right) \sqrt{n} \leq\left|P_{n}(z)\right| \leq\left(1+\varepsilon_{n}\right) \sqrt{n} .
$$

However, Kahane's proof is probabilistic and it is not known how to construct such polynomials. It is not even known how to construct polynomials which satisfy

$$
A \sqrt{n} \leq\left|P_{n}(z)\right| \leq B \sqrt{n}
$$

for some pair of absolute constants $0<A<B<\infty$.

Received by the editors September 24, 1990.

1980 Mathematics Subject Classification (1985 Revision). Primary 42A05.

Key words and phrases. Trigonometric polynomials, extremal problems, unimodular polynomials. The author is also with the MITRE Corporation, McLean, VA. 
The upper bound inequality is not so hard to deal with. Beller [Be] gives polynomials $P_{n}$ which satisfy

$$
\left|P_{n}(z)\right| \leq 1.717 \sqrt{n} \quad \text { for }|z|=1
$$

for $n$ sufficiently large. It is even possible to make the coefficients \pm 1 . The Rudin-Shapiro polynomials $[R, S]$ satisfy

$$
\left|P_{n}(z)\right| \leq \sqrt{2} \sqrt{n} \quad \text { for }|z|=1
$$

but are defined only for $n=2^{k}$. They can be concatenated to give

$$
\left|P_{n}(z)\right| \leq(\sqrt{2} /(\sqrt{2}-1)) \sqrt{n} \quad \text { for }|z|=1
$$

for arbitrary $n$.

Keeping $P_{n}(z)$ bounded away from 0 is a much more delicate matter. No example is known of polynomials $P_{n}(z)=\sum_{m=1}^{n} a_{m, n} z^{m}$ satisfying

(1) $A\left\|P_{n}\right\|_{\infty} \leq\left|P_{n}(z)\right|$ for $|z|=1$.

(2) $\left|a_{m, n}\right|=1$ for $n=1,2, \ldots ; m=1, \ldots, n$.

In [BeN] Beller and Newman give a construction of polynomials $P_{n}$ with coefficients $\left|a_{m, n}\right| \leq 1$ which satisfy (1) with a constant $A$ of about 0.0236 . In this paper we give a construction based on a different idea which yields a constant $A$ of about 0.47 .

Theorem. Let $N$ be an even integer greater than 2 . There exists a trigonometric polynomial

$$
P_{N}(x)=\sum_{k=-N}^{N^{2}} a_{k} e^{i k x}
$$

satisfying

(1) $\left|a_{k}\right| \leq 1$ for $k=-N, \ldots, N^{2}$,

(2) $0.47 N \leq \mid P_{N}(x) ! \leq N$ for all real $x$.

Proof. Let

$$
K_{N}(x)=\frac{1}{N}\left(\frac{\sin N x / 2}{\sin x / 2}\right)^{2} .
$$

Then the Fourier transform is

$$
\widehat{K}_{N}(n)=\left(1-\frac{|n|}{N}\right) \quad \text { for } 0 \leq|n| \leq N
$$

and $\widehat{K}_{n}(n)=0$ otherwise. Let $\sigma$ be the permutation of $(0,1, \ldots, N-1)$ defined by

$$
\sigma=(0,2,4, \ldots, N-2, N-1, N-3, \ldots, 3,1) .
$$

Note that $\sigma^{-1}$ is given by

$$
\sigma^{-1}=(0, N-1,1, N-2,2, N-3, \ldots, N / 2) .
$$

Let $\varepsilon(n)$ for $n=0, \ldots, N-1$ be defined by

$$
\varepsilon(n)= \begin{cases}1 & \text { for } n=0, \ldots,(N / 2)-1, \\ -1 & \text { for }(N / 2), \ldots, N-1 .\end{cases}
$$


Let

$$
f_{k}(x)=\varepsilon(k) e^{i \sigma(k) N x} K_{N}\left(x-\frac{2 \pi k}{N}\right)
$$

Let

$$
P_{N}=\sum_{k=0}^{N-1} f_{k}
$$

We will first show that $\left\|\widehat{P}_{N}\right\|_{\infty} \leq 1$. Note that

$$
\hat{f}_{k}(n)=\varepsilon(k) e^{-2 \pi i k n / N} \widehat{K}_{N}(n-\sigma(k) N) .
$$

Denote by $A_{j}$ the arithmetic progression $j N, j N+1, \ldots,(j+1) N-1$. Then $\hat{f}_{k}$ is supported on the set $A_{\sigma(k)-1} \cup A_{\sigma(k)}$. This means that on any $A_{j}$ at most two of the $\hat{f}_{k}$ are nonvanishing. In particular,

$$
\widehat{P}_{N}(n)= \begin{cases}\hat{f}_{\sigma^{-1}(0)}(n) & \text { for } n \in A_{-1} \\ \hat{f}_{\sigma^{-1}(j)}(n)+\hat{f}_{\sigma^{-1}(j+1)}(n) & \text { for } n \in A_{j}, j=0, \ldots, N-2 \\ \hat{f}_{\sigma^{-1}(N-1)}(n) & \text { for } n \in A_{N-1}\end{cases}
$$

Since

$$
\left|\hat{f}_{\sigma^{-1}(j)}(n)\right|=\left(1-\frac{|n-j N|}{N}\right),
$$

it follows that $\left|\widehat{P}_{N}(n)\right| \leq 1$ for all $n$.

We next show that $\left\|P_{N}\right\|_{\infty}=N$. Let

$$
S_{N}(x)=\sum_{k=0}^{N-1} K_{N}\left(x-\frac{2 \pi k}{N}\right) .
$$

Then $\left|P_{N}(x)\right| \leq S_{N}(x)=K_{N} * \mu(x)$ where $\mu=\sum_{k=0}^{N-1} \delta_{2 \pi k / N}$. Since supp $\widehat{K}_{N} \subset$ $\{-N, \ldots, N\}$ and $\operatorname{supp} \hat{\mu}=N Z$ it follows that $\widehat{S}_{N}=\widehat{K}_{N} \hat{\mu}=\delta_{0}$ which shows that $S_{N}$ is a constant. Evaluating $S_{N}(0)=K_{N}(0)=N$ gives $\left\|P_{N}\right\|_{\infty} \leq N$. But $P_{N}(0)=N$ so that $\left\|P_{N}\right\|_{\infty}=N$.

Finally, we estimate $\min \left|P_{N}(x)\right|$. Suppose $x \in I_{k_{0}}$ where $I_{k}$ is the interval defined by $I_{k}=[2 \pi k / N, 2 \pi(k+1) / N)$. Let

$$
\begin{aligned}
R_{N, N-1} & =f_{N-1}+f_{0}, & & \\
R_{N, k_{0}} & =f_{k_{0}}+f_{k_{0}+1} & & \text { for } 0 \leq k_{0}<N-1, \\
Q_{N, k_{0}} & =P_{N}-R_{N, k_{0}} & & \text { for } 0 \leq k_{0} \leq N-1 .
\end{aligned}
$$

For $x \in I_{k_{0}}$

$$
\left|R_{N, k_{0}}(x)\right|-\left|Q_{N, k_{0}}(x)\right| \leq\left|P_{N}(x)\right| \leq\left|R_{N, k_{0}}(x)\right|+\left|Q_{N, k_{0}}(x)\right| .
$$

We will estimate $\left|R_{N, k_{0}}(x)\right|$ and $\left|Q_{N, k_{0}}(x)\right|$ on $I_{k_{0}}$. Write

$$
\begin{aligned}
\left|R_{N, k_{0}}(x)\right| & =\left|f_{k_{0}}+f_{k_{1}}\right| \\
& =\left|K_{N}\left(x-\frac{2 \pi k_{0}}{N}\right)+\eta e^{i \beta N x} K_{N}\left(x-\frac{2 \pi k_{1}}{N}\right)\right|
\end{aligned}
$$


where $\eta=\varepsilon\left(k_{1}\right) / \varepsilon\left(k_{0}\right)$ and $\beta=\sigma\left(k_{1}\right)-\sigma\left(k_{0}\right)$. The definitions of $\sigma$ and $\varepsilon$ insure that $\beta$ is either \pm 1 or \pm 2 and that

$$
\eta= \begin{cases}+1 & \text { when } \beta= \pm 2 \\ -1 & \text { when } \beta= \pm 1\end{cases}
$$

Consider first the case $k_{0}=N-1$. Then $k_{1}=0, \eta=-1, \beta=-1$. Using (1) we have

(2)

$$
\left|R_{N, N-1}(x)\right|=\left[K_{N}^{2}\left(x+\frac{2 \pi}{N}\right)-2 \cos N x K_{N}\left(x+\frac{2 \pi}{N}\right) K_{N}(x)+K_{N}^{2}(x)\right]^{1 / 2} .
$$

Also using the earlier result that $S_{N}(x)=N$ for all $x$ gives

$$
\begin{aligned}
\left|Q_{N, N-1}(x)\right| & \leq \sum_{k=1}^{N-2} K_{N}\left(x-\frac{2 \pi k}{N}\right)=S_{N}(x)-K_{N}(x)-K_{N}\left(x+\frac{2 \pi}{N}\right) \\
& =N-K_{N}(x)-K_{N}\left(x+\frac{2 \pi}{N}\right) .
\end{aligned}
$$

Tedious but elementary calculations can be used to find the mimimum of

$$
\begin{aligned}
& {\left[K_{N}^{2}\left(x+\frac{2 \pi}{N}\right)-2 \cos N x K_{N}\left(x+\frac{2 \pi}{N}\right) K_{N}(x)+K_{N}^{2}(x)\right]^{1 / 2}} \\
& \quad-N+K_{N}(x)+K_{N}\left(x+\frac{2 \pi}{N}\right)
\end{aligned}
$$

on the interval $[2 \pi(N-1) / N, 2 \pi]$. This minimum is found to exceed $0.619 N$. Hence

$$
0.619 N \leq\left|P_{N}(x)\right| \leq N \text { for } x \in I_{N-1} .
$$

If $k_{0}=(N / 2)-1$ then $\eta=-1$ and $\beta=1$. A similar analysis as in the previous case gives the same bounds on $\left|P_{N}(x)\right|$ for $x \in I_{(N / 2)-1}$.

Next consider $k_{0} \in\{0, \ldots,(N / 2)-2\} \cup\{(N / 2), \ldots, N-2\}$. Then $\eta=1$ and $\beta= \pm 2$. Using (1) we find

$$
\begin{aligned}
\mid R_{N}, k_{0} & (x) \mid=\left[K_{N}^{2}\left(x-\frac{2 \pi k_{0}}{N}\right)+K_{N}^{2}\left(x-\frac{2 \pi\left(k_{0}+1\right)}{N}\right)\right. \\
& \left.+2 \cos 2 N x K_{N}\left(x-\frac{2 \pi k_{0}}{N}\right) K_{N}\left(x-\frac{2 \pi\left(k_{0}+1\right)}{N}\right)\right]^{1 / 2} .
\end{aligned}
$$

As before we can write

$$
\left|Q_{N, k_{0}}(x)\right| \leq N-K_{N}\left(x-\frac{2 \pi k_{0}}{N}\right)-K_{N}\left(x-\frac{2 \pi\left(k_{0}+1\right)}{N}\right) .
$$

Again, tedious but elementary calculations show that the minimum for $x \in I_{k_{0}}$ of

$$
\begin{aligned}
& {\left[K_{N}^{2}\left(x-\frac{2 \pi k_{0}}{N}\right)+K_{N}^{2}\left(x-\frac{2 \pi\left(k_{0}+1\right)}{N}\right)\right.} \\
& \left.+2 \cos 2 N x K_{N}\left(x-\frac{2 \pi k_{0}}{N}\right) K_{N}\left(x-\frac{2 \pi\left(k_{0}+1\right)}{N}\right)\right]^{1 / 2} \\
& \quad-N+K_{N}\left(x-\frac{2 \pi k_{0}}{N}\right)+K_{N}\left(x-\frac{2 \pi\left(k_{0}+1\right)}{N}\right)
\end{aligned}
$$


exceeds $0.47 N$. Therefore

$$
\begin{gathered}
0.47 N \leq\left|P_{N}(x)\right| \leq N \text { for } x \in I_{k_{0}} . \\
\text { REFERENCES }
\end{gathered}
$$

[B] E. Beller, Polynomial extremal problems in $L^{p}$, Proc. Amer. Math. Soc. 29 (1971), 249-259.

[ BeN] E. Beller and D. J. Newman, The minimum modulus of polynomials, Proc. Amer. Math. Soc. 45 (1974), 463-465.

[By] J. S. Byrnes, On polynomials with coefficients of modulus one, Bull. London Math. Soc. 9 (1977), 177-176.

[ E] P. Erdös, Some unsolved problems, Michigan Math. J. 4 (1957), 291-300.

[Ka] J.-P. Kahane, Sur les polynomes a coefficients unimodulaires, Bull. London Math. Soc. 12 (1980), 321-342.

[Ko] T. Körner, On a polynomial of J. S. Byrnes, Bull. London Math. Soc. 12 (1980), 219-224.

[L] J. E. Littlewood, On the mean values of certain trigonometrical polynomials, J. London Math. Soc. 36 (1961), 307-334.

[ R] W. G. Rudin, Some theorems on Fourier coefficients, Proc. Amer. Math. Soc. 10 (1959), 855-859.

[S] H. S. Shapiro, Extremal problems for polynomials and power series, Thesis, M.I.T., 1951.

Department of Mathematics, Georgetown University, Washington, DC 20057 